\title{
Development of Functional Agent Release Fabric Using B-Cyclodextrin Inclusion Compound
}

\author{
Subhas Ghosh, Roopkatha Pallye \\ College of Technology, Eastern Michigan University \\ Ypsilanti, Michigan, USA
}

\begin{abstract}
The $\beta$ - Cyclodextrin inclusion compounds were attached to the 100\% cotton and 50/50 blend cotton/polyester fabrics using Sol-gel, prepared from 3-Glycidoxypropyltrimethoxysilane (GPTMS) and Tetraethoxy orthosilicate (TEOS). The cotton and polyester/cotton fabrics were treated with Sol-gel, which was attached with cotton cellulose through covalent bonding, while in the polyester portion attaches to the Sol-gel through a secondary bonding. $\beta$ - Cyclodextrin molecules were held in place in the microporous structure of a Sol-gel-network and thus attached to the fabric. Aromatherapy oil, having eucalyptus, lavender and lemon fragrances, were applied to the treated fabrics by a selected application method. The durability of these fragrances on fabrics was rated by four judges over six weeks; and up to six washings. The scent ratings decreased over six weeks and six washing; however, the fabrics continued to have a decreased fragrance after, the six weeks. $\beta$ - Cyclodextrin was also attached to the textile fabric by esterification reaction with citric acid in the presence of the catalyst sodiumdihydrogenphosphate. Cross-linking occurs because of the presence of three carboxyl groups in citric acid that are able to bind with the hydroxyl groups of $\beta$-Cyclodextrin and cotton cellulose. As a consequence of crosslinking between the citric acid, $\beta$ - Cyclodextrin and cellulose in fabric, the $\beta$ - Cyclodextrin inclusion complex gets firmly grafted to the textile fabric. The active agent used in the inclusion complex was Thyme (Thymus vulgaris) essential oil which has a strong smell/fragrance. Over a period of time and subsequent wash-cycles, the retention of smell was determined by employing a subjective rating technique. Thymol present in Thyme essential oil applied on the fabric was also determined by colorimetric quantitative estimation.
\end{abstract}

Keywords: $\beta$ - Cyclodextrin, Sol-gel, Aromatherapy, Essential oils, Cross-linking, Citric acid

\section{Introduction}

In the emerging trends of the textile industry, the incorporation of cosmetics/medicine/aroma in textile fabrics has been expanded with the development of sustainable time- release active agents such agents as perfume, odor-trapping components, medicines, anti-oxidants, moisturizer and regenerators. This study develops an active-agent releasing finish on cellulosic fabric and cellulosic blend fabric. Aromatherapy is the use of concentrated essential oils extracted from herbs, flowers and other plants to treat various types of ailments. Brown [1] reported the effects of individual fragrances on human health. Essential oils, like all organic compounds, are made up of complex mixtures of several hydrocarbons such as terpenes, alcohols, esters, aldehydes, ketones, oxides and phenols. Even a simple essential oil may contains 80 - 300 different chemical constituents as reported by $\mathrm{Hu}$ et al. [2]. Authors described the creation of a perfume release fabric using rose fragrance nano-capsules directly on finished cotton fabrics. The authors claimed an excellent sustained fragrance release property. Wang and Chen [3] used aromatherapy oil on textile fabric after treating the fabric with $\beta$ Cyclodextrin, where the inclusion compound was applied to the fabric using the traditional pad method. Essential oil has been used alone or along with Western-style medical treatment in many different cultures [4]. Our investigation is based on the study of the chemical agent releasing textile fabric, where aromatherapy essential oils were used as the releasing agent from the $\beta$-Cyclodextrin (CD) inclusion complex. The $\beta$-Cyclodextrin is a cyclic oligosaccharide compound of six glucose units linked by $\alpha-1,4$ glycoside bonds. They are reported to be produced by the enzymatic degradation of starch by means of $\alpha$-glucosidase or $\alpha$-amylase [5]. It has a conical conformation with a height approximately $0.78 \mathrm{~nm}$ and diameter 0.57 to $0.95 \mathrm{~nm}$. Each Cyclodextrin has a hydrophobic cavity which act as a host for the aromatherapy essential oils. This cavity that can host the $\beta$-Cyclodextrin is mostly used on textiles because of ease of production, price and size of the cavity. A fuller schematic structure of the Cyclodextrin is depicted in Figure 1. [6]

All glucopyranose units in the torus-like ring are reported to possess the thermodynamically favored chair conformation because all substituent are in the equatorial position. Hydroxyl groups on the outside of the Cyclodextrin 
ensure good water solubility. However, the cavity of the ring is lined with hydrogen atoms of C3, by the glycoside oxygen bridges and hydrogen atoms of $\mathrm{C} 5$ making the inner side of such ring appreciably hydrophobic. The C-2-OH group of one glucopyranoside unit can form a hydrogen bond with the $\mathrm{C}-3-\mathrm{OH}$ group of the adjacent glucopyranose unit.

Cyclodextrin behaves more or less like rigid compounds. The Cyclodextrin complex formation is a dimensional fit between the host cavity and guest molecule (essential oil). The lipophilic cavity of Cyclodextrin molecules provides a microenvironment into which appropriately sized non-polar moieties can enter to form inclusion complexes. No covalent bonds are broken or formed during the formation of the inclusion complex. The driving force of a complex formation is the release of enthalpy-rich liquid (water) molecules from the cavity. The binding strength depends on how well the host-guest complex fits together and on specific local interactions between surface atoms. Complexes can be formed either in solution or in the crystalline state and a suitable solvent including water.

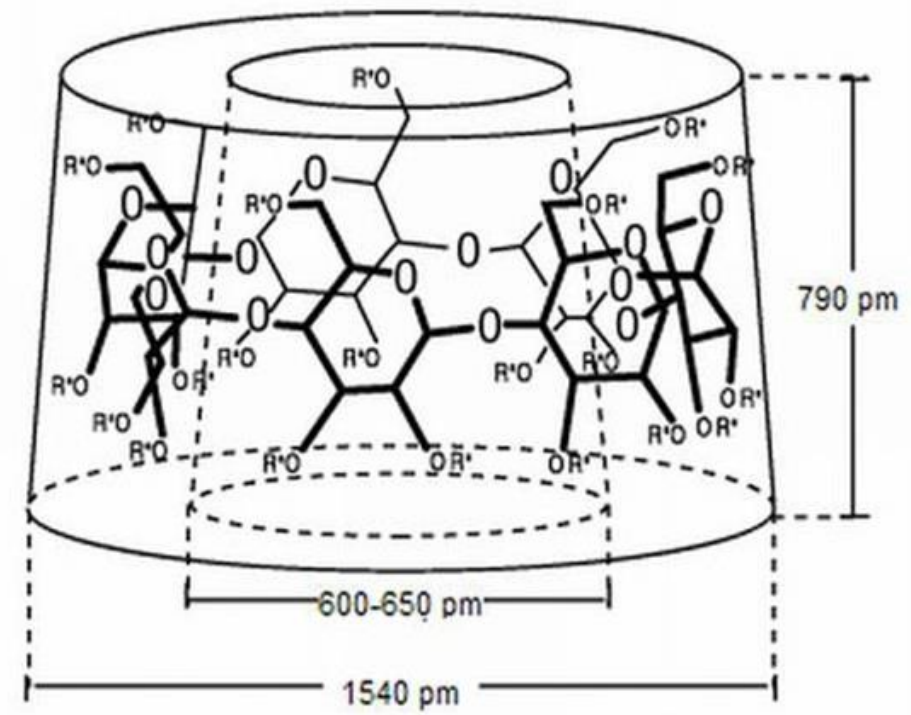

Fig. 1: Schematic structure of $B$-Cyclodextrin [6].

Sols are the dispersion of colloidal particles in liquid having a diameter ranging from $1 \mathrm{~nm}$ to $100 \mathrm{~nm}$. Gels are interconnected polymer chains with rigid networks and pores of sub-nanometer_dimensions, whose average length is greater than a nanometer [7]. The sol-gels used in this investigation were prepared from two precursors, 3glycidyloxypropyltrimethodoxysilane (GPTMS) and Tetraethoxy orthosilicate (TEOS). TEOS makes a sol and forms a gel when cross-linked with GPTMS. In the first experiment cotton and polyester/cotton fabrics were treated with sol-gel, which attaches with cotton cellulose through covalent bonding [8]. The durable attachment of sol-gel to polyester is attributed to the secondary bonding of sol-gel to polyester, and cross-linking during curing that forms a film on the fabric surface. $\beta$-Cyclodextrin molecules are held in place in the micro- porous structure of a sol-gel-network. Essential oils are introduced into the cavities of $\beta$-Cyclodextrin inclusion complex. The $\beta$-Cyclodextrin has also been grafted onto the cotton cellulose fabric in another experiment by introducing cross-linking between the $\beta$-Cyclodextrin and textile substrate using citric acid (a poly carboxylic acid) in the presence of Sodiumdihydrogenphosphate catalyst. Figure 2 illustrates the schematic of the cross-linking process. The presence of thymol in thyme essential oil applied on the fabric was ascertained by colorimetric estimation of thymol. This was done separately in selected combinations, to produce an easy- tomanufacture, cost-effective, and environmentally friendly active agent-releasing cellulosic fabric that can resist multiple wash-cycles. The active agent used in the inclusion complex was Thyme (Thymus vulgaris) essential oil, which has a strong smell/fragrance. 




Fig. 2: Schematic representation of the cross-linking between Cellulosic fabric, Citric acid and $\beta$-Cyclodextrin.

\section{Materials and Methods}

\subsection{Using Sol-gel technique}

Wang and Chen's [2] procedure was modified to create a new synthesis procedure in this study.

GPTMS, TEOS, HCL, dimethyl sulfoxide, Dibutyl amine, $\beta$-Cyclodextrin and methanol were obtained from SigmaAldrich and used without further purification. Aromatherapy essential oils were purchased from Mountain Rose Herbs Company. Cotton (100\%) and polyester/cotton (40/60) blend fabrics were used. The cotton fabric weight was 3.5 oz. /yard ${ }^{2}$, which was plain weave with a 87 X 62 (ends X picks per inch) construction, while the polyester/cotton (40/60) blend fabric weighed $5 \mathrm{oz} . / \mathrm{yard}^{2}$ with a100 X 47 (ends X picks per inch) construction. The specimens from both fabrics were prepared by washing them with $2 \mathrm{~g}$ of disodium carbonate in $1 \mathrm{~L}$ water for $1 \mathrm{~h}$ at $75^{\circ} \mathrm{C}$. The fabric was then washed and dried.

\subsubsection{Preparation of TEOS Solution}

One gram of $\mathrm{HCl}$ was mixed well in a beaker with $99 \mathrm{~g}$ of DI water. Sixty-five gram (0.3 mole) of TEOS were poured into a plastic beaker and the $\mathrm{HCl}$ solution was added while stirring at a high speed and maintaining the average $\mathrm{pH}$ at 3.5, varied within the range of 3.0 from 4.0. The solution was stirred for 8 hrs.

\subsubsection{Preparation of the $\beta$ - Cyclodextrin Solution and Sol-gel preparation using GPTMS and TEOS}

$\beta$ - Cyclodextrin (36g or 0.3 moles) was mixed with $130 \mathrm{~g}$ of dimethyl sulfoxide anhydrous and stirred until the solution became clear. The stirring continued for one additional hour after the solution became clear.

In order to open GPTMS' glycedal ring, $0.1 \mathrm{~g}$ of Dibutyl amine was mixed with $70.8 \mathrm{~g}$ (0.3 moles) of GPTMS, and stirred for $10 \mathrm{~min}$. The TEOS solution was then added and the solute was reduced to $60 \%$ with methanol and distilled water, followed by stirring for $15 \mathrm{~min}$. The sol-gel is formed by a hydrolysis reaction, followed by a condensation reaction. The schematic structure of a sol-gel formation is shown in Figure 3, where some free Si-O-Et or Si-O-Me will eventually hydrolyze/condense to form epoxy-functional silicate particles. $\beta$-Cyclodextrin solution was then added to the mixture and stirred for $1 \mathrm{hr}$.

\subsubsection{Application on Textile Fabric}

Both cotton and polyester/cotton fabric specimens were dipped into the solution while stirring, and the specimen processed through the padder to evenly distribute the solution under pressure $\left(0.18 \mathrm{lb} / \mathrm{inch}^{2}\right)$. Three specimens were prepared for each sample, and each specimen went through the application process twice. The wet pick-up for cotton fabric varied from $123 \%-125 \%$ and in the range of $127 \%-134 \%$ for polyester/cotton fabric. The cotton fabric weight was 3.5 oz. /yard ${ }^{2}$, while the polyester/cotton fabric weighed $5 \mathrm{oz}$. /yard ${ }^{2}$. The specimens were dried in an oven at $160^{\circ} \mathrm{C}$ for an hour. The dry add-on was measured for both specimens, which revealed an average of $24.7 \%$ and $31 \%$ (w/w) dry add-ons for the cotton and the poly/cotton fabric, respectively. A higher add-on for the polyester/cotton fabric can be attributed to $42 \%$ higher fabric weight. 


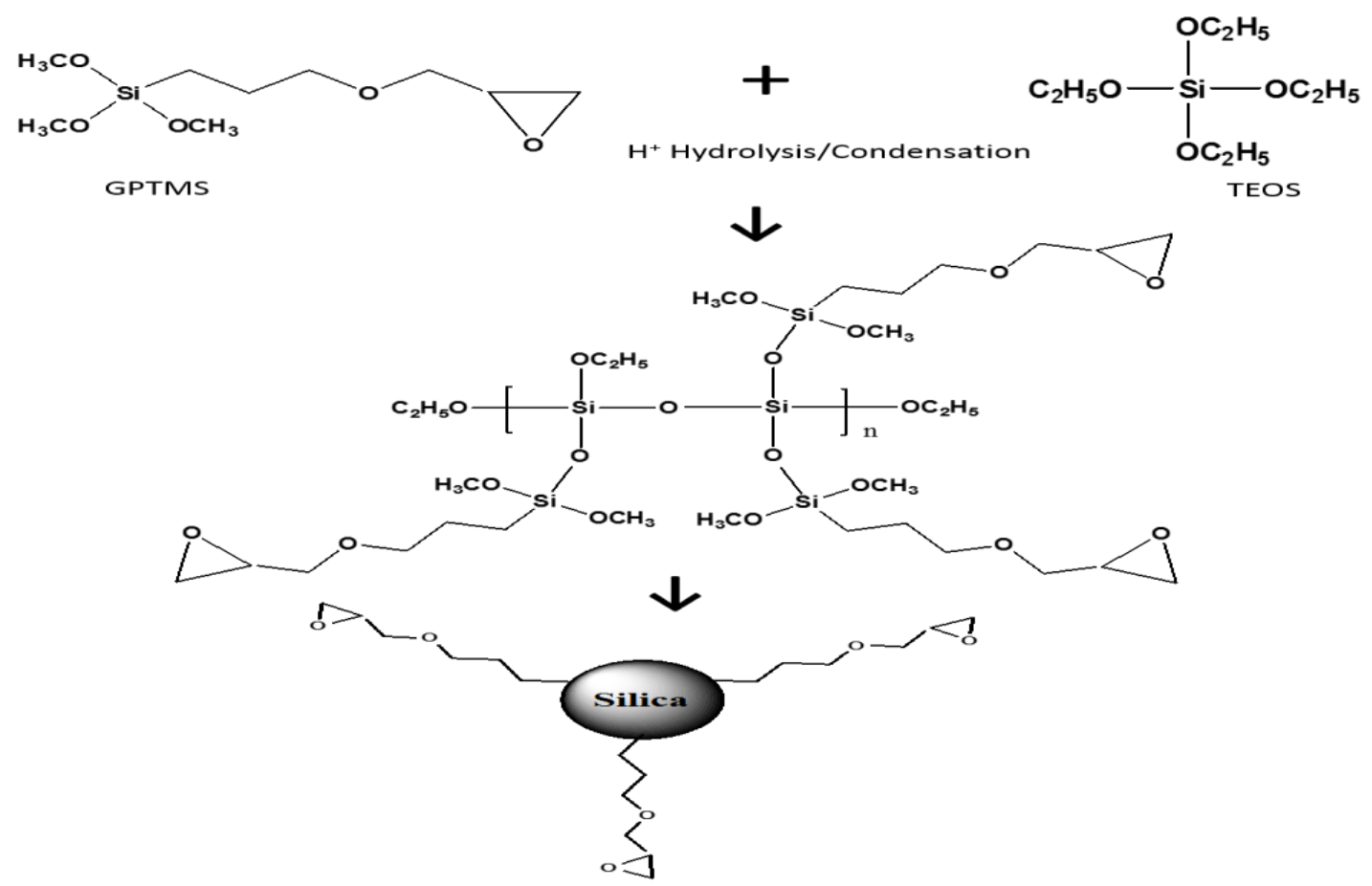

Fig. 3: Schematic structure of sol-gel formation.

\subsubsection{Essential Aromatherapy Oil Application using on samples prepared by Sol-gel and CDs}

The dried specimen fabrics were force-sprayed with undiluted aromatherapy essential oils until the specimens were completely soaked. It was observed that spray application yielded better results in the scent intensity than the dip and padding application process. The spraying force helped the essential oil to penetrate the cavities of the $\beta$-Cyclodextrins. All of the specimens were dried overnight at ambient conditions. Formation of inclusion complex is illustrated in Figure 4 [5].



Fig. 4: Formation of Inclusion Complex [5].

\subsection{Using Citric Acid and CDs Cross-linking Technique}

Several samples of cotton fabric were padded at $100 \%$ expression separately with aqueous solutions containing $10 \mathrm{~g} / \mathrm{l}$ citric acid, $10 \mathrm{~g} / 1$ catalyst, $20 \mathrm{~g} / 1$ ethanol solution of thyme essential oil, and $10 \mathrm{~g} / 1 \beta$-Cyclodextrin in five different combinations. Fabric Sample 1 and 4 had all the constituents; sample 2 had all the constituents except that the amount of $\beta$ Cyclodextrin was increased to $20 \mathrm{~g} / \mathrm{l}$. Sample 3 didn't have any $\beta$-Cyclodextrin, and sample 5 had neither Cyclodextrin nor citric acid-catalyst system. For sample 4 only, the fragrance was applied by spraying after pad-dry-cure. For all other samples, the fragrance was applied by dipping the samples into the thyme essential oil solution in ethanol before padding. 
The padded fabrics were thereafter dried at $95^{\circ} \mathrm{C}$ for 5 minutes and subsequently cured at $140^{\circ} \mathrm{C}$ for 5 minutes. The cured fabrics were washed in cold water and dried in air.

\section{Results and Discussion}

\subsection{Fabric samples treated with Sol-gel and CDs}

The fragrance durability of cotton and polyester/cotton fabrics is shown in Table 1 . The average rating was based on the 4 individual specimens for each fabric type scent and the 4 judges. A 0 value means no fragrance was detected, while a 5 value indicates very high fragrance. A 0.25 standard error of estimate of the judge's ratings was determined at a $95 \%$ level of confidence. In most cases, the judges rated the fragrance intensity very similarly. Fragrance release from the fabrics is influenced by the presence of $\beta$-Cyclodextrin and the solvent used in the aromatherapy oils. In general, the rating points are higher for the polyester/cotton fabric than for the cotton fabric for all scents. This phenomenon can be attributed to a higher add-on of $\beta$-Cyclodextrin onto the polyester/cotton fabric, which causes it to hold more aromatherapy essential oils. The first rating was given to the fabrics the day after the scents were applied, and the final rating was given after a period of 6 weeks and 6 washes. As expected, with time, a general decreasing trend of ratings was observed for all fragrances on both fabrics. The rating values do not show a significant difference in the scents' intensity between the different types of scents used. The presence of the scents was greatly reduced after 6 weeks and 6 washes; however, it does not disappear completely. For polyester/cotton fabric, the fragrances of lemon and lavender were clearly evident after 6 weeks.

Table 1: Average scent intensity rating by judges.

\begin{tabular}{|c|c|c|c|c|c|c|}
\hline \multirow[b]{2}{*}{$\begin{array}{l}\text { Evaluation } \\
\text { day }\end{array}$} & \multicolumn{2}{|c|}{ Eucalyptus } & \multicolumn{2}{|c|}{ Lemon } & \multicolumn{2}{|c|}{ Lavender } \\
\hline & Polyester/Cotton & Cotton & Polyester/cotton & Cotton & Polyester/cotton & Cotton \\
\hline & 4.25 & 3.25 & 5.00 & 4.25 & 4.25 & 3.25 \\
\hline 5 (1wash) & 3.50 & 3.38 & 4.38 & 3.13 & 4.75 & 3.13 \\
\hline 10 ( 2 wash) & 3.75 & 2.25 & 4.50 & 2.50 & 4.25 & 2.75 \\
\hline 15 (3 wash) & 3.25 & 2.00 & 3.75 & 2.50 & 4.00 & 2.25 \\
\hline 20 (4 wash) & 2.75 & 2.00 & 3.50 & 2.00 & 3.25 & 2.00 \\
\hline 25 (5 wash) & 2.00 & 1.63 & 2.25 & 1.75 & 2.63 & 1.63 \\
\hline 30 (6 wash) & 0.50 & 1.00 & 1.88 & 0.50 & 1.25 & 0.75 \\
\hline
\end{tabular}

\subsection{Fabric Samples Cross-linked with Citric acid and CDs}

The main fragrance compound thymol, present in the thyme essential oil $60-66 \%$ by volume, is a monoterpenoid phenol derivative. Diazonium salts readily react with phenols to form highly coloured derivatives known as azocompounds, in slightly alkaline condition. To determine the concentration of thymol in the different thymol finished fabrics, thymol was extracted from the fabric using ethanol. The ethanol extracts were used to react with $10 \mathrm{ml}$ of 2 4DNPH, periodate and $\mathrm{NaOH}$ solution allowing colour to develop. The concentrations of thymol extracted from the treated fabrics were then determined using a calibration curve drawn on the UV-Vis Spectrophotometer by measuring absorbance of thymol solution of known concentrations at $4 \mathrm{~nm}$ wavelength. The smell retention was checked through a period of one month, at 15days interval through 3 wash cycles, and thymol concentration was determined after 30days. Samples' fragrance durability was also subjectively rate by judges using 1 to 5 as described earlier. Subjective and quantitative data are presented in Table 2 . 
Table 2: Quantitative and Subjective Rating Data for Cyclodextrin and Citric Acid Treated Fabrics.

\begin{tabular}{|c|c|c|c|}
\hline & Absorbance & Concentration & Subjective Rating \\
\hline $\begin{array}{c}\text { Fabric } \\
\text { Treated with } \\
10 \mathrm{~g} / \mathrm{l} \mathrm{Citric} \mathrm{acid} \\
+10 \mathrm{~g} / 1 \mathrm{NaH} \mathrm{PO}_{4} \text { catalyst } \\
+10 \mathrm{~g} / \mathrm{l} \mathrm{CD}+ \\
20 \mathrm{~g} / \mathrm{l} \mathrm{fragrance}\end{array}$ & 0.825 & 0.198 & 3 \\
\hline $\begin{array}{c}\text { Fabric } \\
\text { Treated with } \\
10 \mathrm{~g} / \mathrm{l} \mathrm{Citric} \mathrm{acid} \\
+10 \mathrm{~g} / 1 \mathrm{NaH} \mathrm{PO}_{4} \text { catalyst } \\
+20 \mathrm{~g} / \mathrm{l} \mathrm{CD}+20 \mathrm{~g} / \mathrm{l} \\
\text { fragrance }\end{array}$ & 0.783 & 0.172 & 4 \\
\hline $\begin{array}{c}\text { Fabric } \\
\text { Treated with } \\
10 \mathrm{~g} / \mathrm{l} \mathrm{Citric} \mathrm{acid} \\
+10 \mathrm{~g} / \mathrm{l} \mathrm{NaH}_{2} \mathrm{PO}_{4} \text { catalyst }+ \\
20 \mathrm{~g} / \mathrm{l} \text { fragrance }\end{array}$ & 0.716 & 0.136 & 2 \\
\hline $\begin{array}{c}\text { Fabric } \\
\text { Treated with } \\
10 \mathrm{~g} / \mathrm{l} \mathrm{Citric} \mathrm{acid} \\
+10 \mathrm{~g} / 1 \mathrm{NaH}_{2} \mathrm{PO}_{4} \text { catalyst } \\
+10 \mathrm{~g} / \mathrm{l} \mathrm{CD}+ \\
20 \mathrm{~g} / \mathrm{l} \text { fragrance(after pad- } \\
\text { dry-cure) }\end{array}$ & 0.956 & 0.207 & 4 \\
\hline $\begin{array}{c}\text { Fabric } \\
\text { Treated with } \\
\text { Only } 20 \mathrm{~g} / \mathrm{l} \text { fragrance }\end{array}$ & 0.429 & 0.121 & 2 \\
\hline
\end{tabular}

The subjective ratings comply with the absorbance-concentration data obtained from the UV-Vis Spectroscopy measurements. The retention of fragrance by the cotton fabric after washing in detergent solution is highest for the sample finished with $10 \mathrm{~g} / \mathrm{l}$ citric acid and $10 \mathrm{~g} / \mathrm{l}$ Cyclodextrin with the fragrance applied after pad-dry-cure. However for fabric finished with $10 \mathrm{~g} / \mathrm{l}$ citric acid and $20 \mathrm{~g} / \mathrm{l}$ Cyclodextrin the fragrance retention value appears to be only marginally higher and is therefore considered to be of little practical significance. The fabric that was not treated with Cyclodextrin and citric acid showed almost no retention of fragrance.

\section{Conclusion}

In this study, $\beta$ - Cyclodextrin inclusion compound was attached to the textile fabric using two different techniques: 1. using Sol-gel to anchor CDs into the micro-pores structure of the Sol-gel and thus attached to the fabric, and 2. attaching Cyclodextrin by cross-linking with citric acid and cotton cellulose. In both cases aromatherapy essential oil was retained by the fabric for at least six weeks, even after several washing. Treated fabric continued to release fragrance even after six weeks. It is evident that the amount of loading essential oils and attachment of Cyclodextrin have influence on the retention of the fragrance.

\section{References}

[1] D. Brown, Aromatherapy. Lincolnwood, Chicago: NTC Publishing Group, 1996, 51-60.

[2] J. Hu, Z. Xiao, S. Ma, M. Wang and Z. Li, "Comparison of compounded fragrance and chitosan nanoparticles loaded with fragrance," Chinese Chem. Eng., vol. 19, no. 3, pp. 523.2, 2011.

[3] X. Wang and S. L. Chen, "Surface treatment of cotton using $\beta$-Cyclodextrins sol-gel method," Applied Surface Science, vol. 252, no. 18, pp. 6348-6352, 2006. 
[4] G. Martin, "Olfactory Remediation: Current Evidence and Possible Applications," Social Science and Medicine, vol. 43, no. 1, pp. 63-70, 1996.

[5] L. Huang, E. Allen and A. Tonelli, "Inclusion compounds formed between Cyclodextrins and nylon 6," Polymer, vol. 40, no. 11, pp. 3211-3221, 1999.

[6] A. Tonelli, "The potential for improving medical textiles with Cyclodextrin inclusion compounds," Journal of Textile and Apparel, Technology and Management, vol 3, no. 2, pp. 1233, 2003.

[7] B. Mohlitig and J. Textor, "sol-gel, prepared from 3-glycidoxypropyltrimethoxysilane and tetraethoxy orthosilicate," $J$ Sol-Gel Sci. \& Technol, vol. 39, pp. 111, 2006.

[8] S. Ghosh and N. Chipot, "Embedding aromatherapy essential oils into textile fabric using Cyclodextrin Inclusion compound," Indian Journal of Fibre \& Textile Research, vol. 40, pp. 140-143, 2015. 